\title{
Heterologous valve implantation in the infra-renal vena cava for treatment of the iliac venous valve regurgitation disease: experimental study
}

Implante de válvula heteróloga na veia cava inferior infra-renal para tratamento da doença de regurgitação valvar venosa ilíaca: estudo experimental

Otoni M. GOMES; Ricardo ZANDIN; João Batista Tomás RODRIGUES; Emerson David MIRANDA; Divino BARROS; Diva Maria de OLIVEIRA

Descriptors: Varicose veins, surgery. Venous insufficiency, surgery. Iliac vein, surgery. Inferior vena cava, surgery.

It was demonstrated in recent studies employing the duplex-scanning technique that iliac venous valve regurgitation was observed in $78 \%$ of patients studied with varicosity in the lower extremities [1].

For the surgical treatment of valve insufficiency in patients with venous diseases of the lower limbs skilled procedures have been performed [2-5], although limited to the femoral and/or greater saphenous veins.

The evidence of simultaneous (bilateral) valve regurgitation disease in both left and right iliac veins sustain this hypothesis for the study of treatment by endovascular (valved stents) or direct surgical implantation of valve prosthesis in the initial segment of the inferior vena cava or in the iliac veins. In this case, the implantation above the ostium of the iliac intern vein preserves the resource of collateral circulation to the opposite side, reducing risk for the treated limb in case of implant obstruction.

In six mongrel dogs, $(15-20 \mathrm{~kg}$ of body weight), under anesthesia with xylazine, thiopental and halothane, the vena cava was exposed below the renal vein by paramedian pararetal intern laparotomy. After systemic heparinization (5 UI/kg body weight) and proximal and distal clamping a segment of a porcine aortic valve cusp preserved in

Faculdade de Medicina do Vale do Aço - UNIVAÇO Gestão CESVALE - Ipatinga - MG

Fundação Cardiovascular São Francisco de Assis - ServCor / Belo

Horizonte - MG, Brazil

Correspondence address: Otoni Moreira Gomes. Rua José do

Patrocínio, 522, Santa Mônica - Belo Horizonte - MG, Brasil.

CEP: 31525-160 Tel / Fax : (31) 3452-7143

E-mailservicor@servicor.com.br 
glutaraldehyde (Comex Ind. Com. Ltda-MG) was implanted through transversal incision. The base of the cusp segment was fixed by the same suture as the venae cavae wall repair.

The result in two initial cases showed after 15 days followup that the preservation of the implanted cusp tissue was without local thrombosis (Figure 1). This confirms the good results reported by FONTAN and BAUDET [6] with implantation of valve bioprosthesis in the inferior and

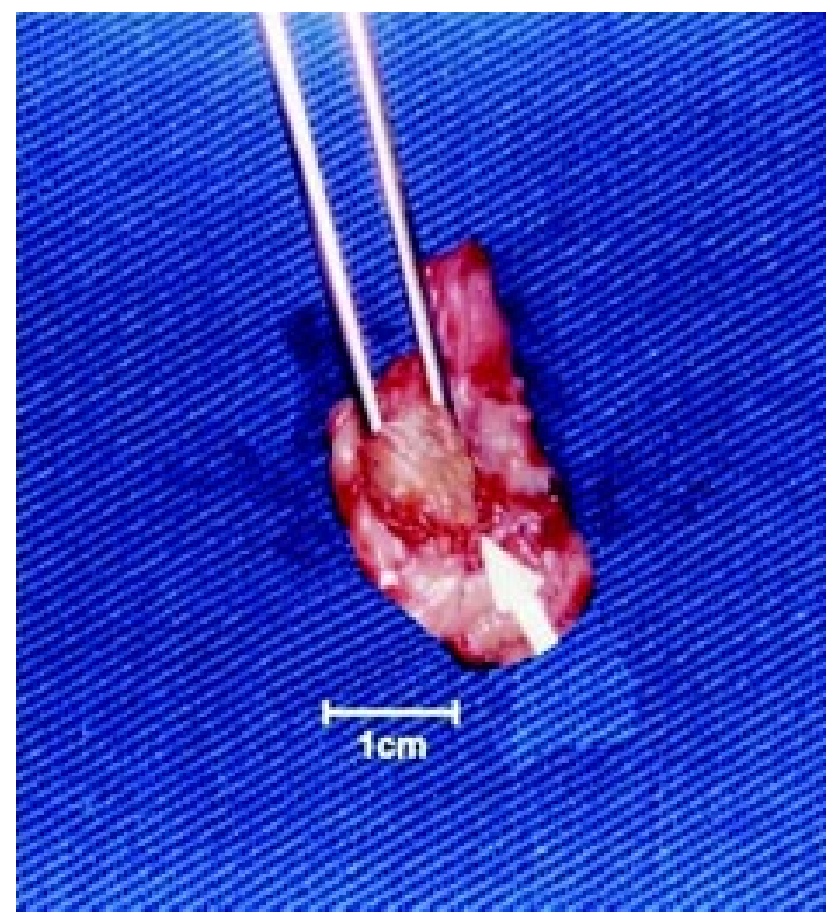

Fig. 1 - Fifteen days follow-up: aortic porcine cusp (arrow) glutaraldheyde preserved implanted in the infrarenal vena cava $(\operatorname{dog} 3)$.

Descritores: Varizes, cirurgia. Insuficiência venosa, cirurgia. Veia Ilíaca, cirurgia. Veia cava inferior, cirurgia.

Demonstrou-se em estudos recentes com técnica Duplex Scanning que a regurgitação valvar venosa ilíaca ocorreu em $78 \%$ dos pacientes estudados com varizes de membros inferiores [1].

Para o tratamento da insuficiência valvar venosa em membros inferiores, operações bem elaboradas têm sido realizadas [2-5], embora limitadas às veias femorais e/ou safenas magnas.

A evidência de doença de regurgitação valvar simultânea em veias ilíacas direitas e esquerdas sustenta a hipótese para estudo de tratamento com implante de bioprótese em veia cava inferior infra-renal ou em veia ilíaca comum. Nestes casos, acima do óstio da veia ilíaca interna, para permitir superior thoracic vena cava for treatment of tricuspid atresia.

Should successful valve implantation in the abdominal infra-renal vena cava be confirmed, in the future, new horizons will be opened for treatment of venous diseases in the lower limbs. This will possibly be through implantation of valved biostents, in the inferior vena cava or in the iliac vein (Figure 2), in cases of bilateral or unilateral commitment, respectively.

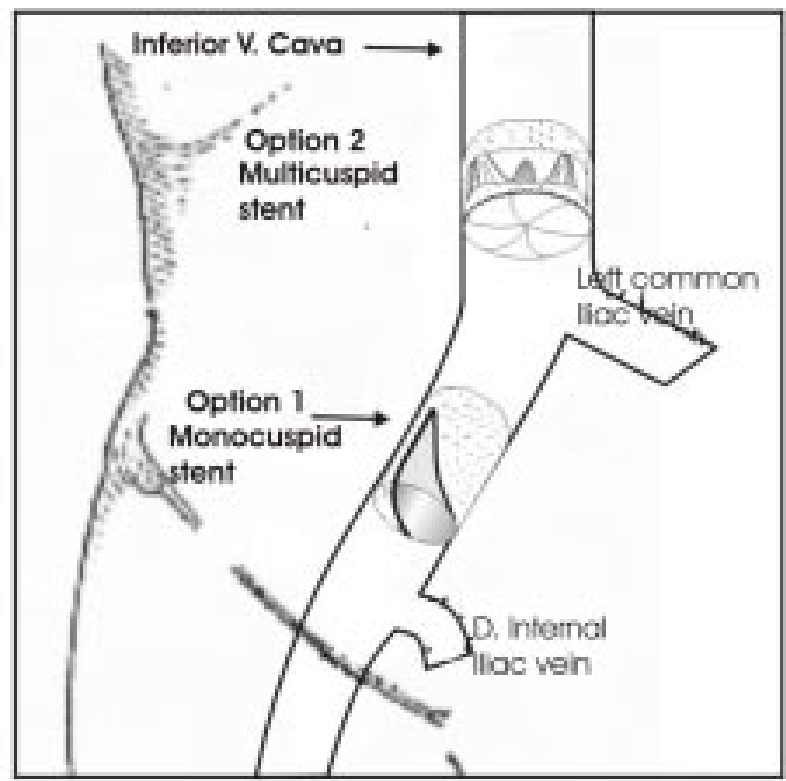

Fig. 2 - Options of valved stent implatation in common iliac or inferior vena cava.

circulação colateral para o lado oposto em caso de obstrução do implante, diminuindo o risco para o membro operado.

Em seis cães mestiços (15 - $20 \mathrm{~kg}$ de peso corpóreo), sob anestesia com xilazina, halotano e thiopental, por meio de incisão abdominal para-retal interna direita, a veia cava infrarenal foi exposta. Após heparinização (5 U.I. / kg de peso corpóreo) e isolamento da veia cava infra-renal por pinçamento proximal e distal, um segmento de cúspide valvar aórtica porcina preservada em glutaraldeído (Comex Ind. Com. Ltda. - MG) foi implantado, por meio da abertura da veia por incisão transversal, sendo a base da cúspide fixada na mesma sutura de síntese da parede venosa.

Em dois casos iniciais, constatou-se a preservação dos segmentos de cúspides implantados, no 150 dia de seguimento pós-operatório, sem trombose local (Figura 1), confirmando resultados satisfatórios descritos por FONTAN 
e BAUDET [6] com implante de bioprótese em veia cava intratorácica, para tratamento da atresia tricúspide.

Caso obtenha-se o sucesso com implante de biopróteses valvares na veia cava inferior infra-renal, novos horizontes serão abertos para o tratamento futuro de doenças venosas em membros inferiores, possivelmente através do implante de biostents valvados na veia cava inferior ou em veia ilíaca (Figura 2), respectivamente, para o acometimento bilateral ou unilateral.

\section{BIBLIOGRAPHIC REFERENCES}

1. Gomes OM, Gomes ES. Iliac valve regurgitation disease. In: X Meeting of the International Society for Heart Research - Latin American Section; 2002; La Plata, Argentina: International Society for Heart Research.
2. Queral LA, Whitehouse Jr. WM, Flinn WR, Neiman HL, Yao JS, Bergan JJ. Surgical correction of chronic deep venous insufficiency by valvular transposition. Surgery 1980; 87: 688-95.

3. Belcaro G. Plication of the sapheno-femoral junction. Phlebology 1991; 6: 159-65.

4. Raju S . Valvuloplasty and valve transfer. Int Angiol 1985; 4: 419-24.

5. Kistner RL. Surgical repair of the incompetent femoral vein valve. Arch Surg 1975; 110: 1336-42.

6. Fontan F, Baudet E. Surgical repair of tricuspid atresia. Thorax 1971; 26:240-8. 